\title{
Arctic terrestrial biodiversity status and trends: A synopsis of science supporting the CBMP State of Arctic Terrestrial Biodiversity Report
}

\author{
Jason J. Taylor, James P. Lawler, Mora Aronsson, Tom Barry, \\ Anne D. Bjorkman, Tom Christensen, Stephen J. Coulson, \\ Christine Cuyler, Dorothee Ehrich, Knud Falk, Alastair Franke, \\ Eva Fuglei, Mark A. Gillespie, Starri Heiðmarsson, Toke Høye, \\ Liza K. Jenkins, Virve Ravolainen, Paul A. Smith, Pawel Wasowicz, \\ Niels Martin Schmidt
}

Published online: 18 January 2020

\begin{abstract}
This review provides a synopsis of the main findings of individual papers in the special issue Terrestrial Biodiversity in a Rapidly Changing Arctic. The special issue was developed to inform the State of the Arctic Terrestrial Biodiversity Report developed by the Circumpolar Biodiversity Monitoring Program (CBMP) of the Conservation of Arctic Flora and Fauna (CAFF), Arctic Council working group. Salient points about the status and trends of Arctic biodiversity and biodiversity monitoring are organized by taxonomic groups: (1) vegetation, (2) invertebrates, (3) mammals, and (4) birds. This is followed by a discussion about commonalities across the collection of papers, for example, that heterogeneity was a predominant pattern of change particularly when assessing global trends for Arctic terrestrial biodiversity. Finally, the need for a comprehensive, integrated, ecosystem-based monitoring program, coupled with targeted research projects deciphering causal patterns, is discussed.
\end{abstract}

Keywords Arctic - Biodiversity monitoring - CAFF . CBMP · Focal ecosystem components - Status and trends

\section{INTRODUCTION}

Temperatures in the Arctic have, generally, increased by more than double the global average over the last two decades (IPCC 2019). This rapid change is expected to result in widespread shifts in the diversity and distribution of animal and plant species (Callaghan et al. 2004; Meltofte 2013) and ultimately the interactions among species (Gilg et al. 2012). Our ability to understand the consequences of these changes, and specifically what is happening, where, and to what extent, depends on the availability of systematically collected monitoring data, access to those data, and a representative geographic and temporal extent of monitoring.

The Circumpolar Biodiversity Monitoring Program (CBMP), an initiative within the Conservation of Arctic Flora and Fauna (CAFF) working group of the Arctic Council, was formed to address the pan-Arctic need for relevant, integrated biodiversity monitoring information. The CBMP is an international network of scientists, managers, conservation organizations, government agencies, and Arctic community experts and leaders that collaborate to develop and implement long-term and comprehensive plans for monitoring the integrity of Arctic biomes: marine (Gill et al. 2011), freshwater (Culp et al. 2012), terrestrial (Christensen et al. 2013), and coastal (Jones et al. 2019). CBMP monitoring plans were developed based on expert opinon using an ecosystem-based approach. Each plan provides a framework for data collection and harmonization across space and time to determine the status and trends of Arctic biodiversity and supporting ecosystems and processes, to identify knowledge gaps and priorities for monitoring, and to provide timely information to managers, decision-makers, and communities within the Arctic and globally.

The CBMP Arctic Terrestrial Biodiversity Monitoring Plan (Christensen et al. 2013), the basis for this special issue, was developed within the context of a broad-scale conceptual model (Beever and Woodward 2011). The model (Fig. 1) was developed to characterize key system relationships, functions, and organization among terrestrial biotic groups and interactions with abiotic components at multiple scales. This model, in part, guided the identification and selection of priority monitoring targets, or focal ecosystems components (FECs), for the terrestrial CBMP. 
This paper is intended as a stand-alone, comprehensive summary of the main findings of individual papers in the special issue, Terrestrial Biodiversity in a Rapidly Changing Arctic. We highlight salient points organized by taxonomic groups: (1) vegetation, (2) invertebrates, (3) mammals, and (4) birds, as identified within the terrestrial conceptual model. We conclude this final paper of the special issue by positing the need for the CBMP to work toward a single, circumpolar, multi-disciplinary, cross-biome monitoring plan that is based on an integrated suite of "core" FECs selected from across the four CBMP ecosystem monitoring plans. These core FECs would serve for holistically assessing and reporting on the state of Arctic biodiversity and biodiversity monitoring.

\section{VEGETATION}

In the CBMP Arctic Terrestrial Biodiversity Monitoring Plan (Christensen et al. 2013), a number of FEC attributes were identified that specifically address vegetation. These include species diversity and composition, phenology, spatial structure, demographics, temporal cycles, health, and productivity and other ecosystem functions and processes. Presented within this issue are complementary approaches to vegetation monitoring, ranging from plotbased measures (Bjorkman et al. 2020) to literature and database analyses (Wasowicz et al. 2020), conceptual discussions (Ravolainen et al. 2020), and pan-Arctic remote sensing derived data analyses (Jenkins et al. 2020). Monitoring attributes describing productivity and phenology is

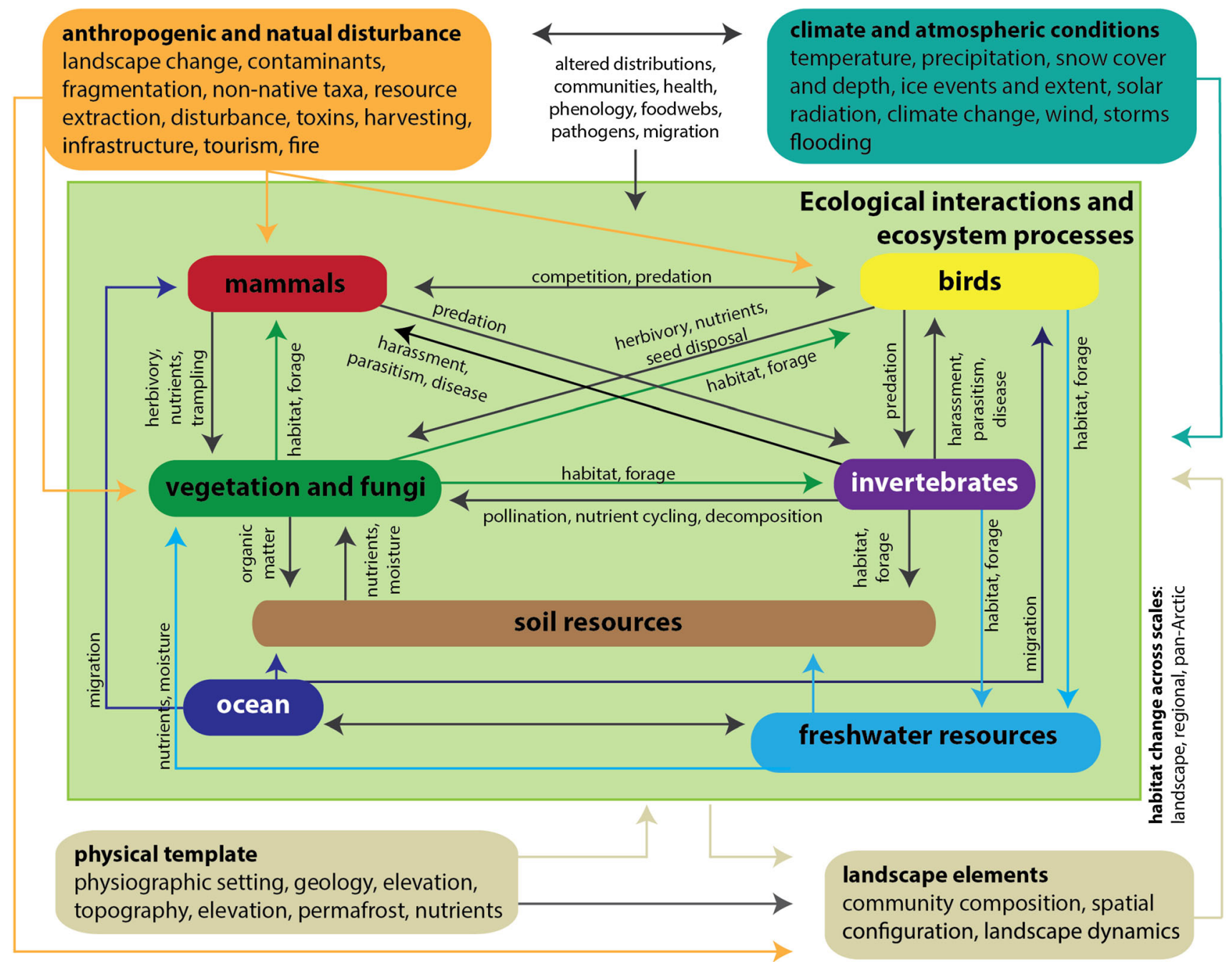

Fig. 1 Conceptual model of the Arctic terrestrial biome, created to support development of the CBMP Arctic Terrestrial Biodiversity Monitoring Plan (Christensen et al. 2013). The model shows key biotic groups (e.g., mammals and invertebrates), relationships among those groups (arrows), and system drivers (e.g., anthropogenic and natural disturbance) 
conducted using both remotely sensed and plot-based observations. In addition, plot-based monitoring provides information on plant abundance, composition, and diversity.

\section{Vegetation: Biodiversity status}

Many of the physical and ecological parameters that drive terrestrial vegetation have experienced significant change over the past decades. For example, seasonal land surface temperature has increased significantly since 2001 (Jenkins et al. 2020). These rapid changes in the physical environment highlight the importance of a systematic approach to monitoring across the Arctic, including ecological responses associated with Arctic vegetation.

The most comprehensively monitored vegetation attribute is phenology, specifically leaf green-up in the spring and leaf senescence in the fall, which has been monitored using both remote sensing (Jenkins et al. 2020) and plotbased (Bjorkman et al. 2020) methods. Both methods identify a slight trend toward earlier green-up over time, as well as in response to experimental warming (Bjorkman et al. 2020). However, trends in green-up varied by bioclimatic subzone, with significant advancing trends identified by remote sensing methods in only two of five Arctic subzones (Circumpolar Arctic Vegetation Map (CAVM) subzones $\mathrm{C}$ and $\mathrm{E}$; Jenkins et al. 2020). In contrast to spring green-up, fall senescence was relatively stable; remote sensing methods did not detect significant trends for any CAVM subzone (Jenkins et al. 2020) and the single plotbased monitoring observation of senescence trends over time identified no significant change.

Plot-based monitoring of flowering time revealed mixed responses; flowering date advanced significantly in six species, did not change in eight species, and was significantly delayed in one species (Bjorkman et al. 2020). Remote sensing methods also identified a significant increase in growing season length, nominally extending the season by approximately 4 days over the study period, in two of five CAVM subzones (subzones B and E; Jenkins et al. 2020). In addition, seasonal vegetation productivity or greenness, measured with the remote sensing metric, Normalized Difference Vegetation Index (NDVI), showed a significant increase across the entire pan-Arctic region since 2001 (Jenkins et al. 2020).

A review of plot-based changes in community composition and abundance (Bjorkman et al. 2020) found large variation among sites and species in the direction and magnitude of change in abundance. Forb, graminoid, and shrub abundance changed significantly (increased or decreased) over time in roughly a third of published studies, while roughly half of the studies identified no significant trends in abundance. In studies investigating the effect of experimental warming on community composition, the majority of the responses exhibited no statistical change, while a small proportion decreased (particularly lichens) and an even smaller proportion increased significantly in abundance.

An additional aspect of changing vegetation composition was addressed in the study by Wasowicz et al. (2020), who investigated trends in exotic species moving into the Arctic. They identified 341 non-native and invasive plant species currently present in the Arctic, a much higher number than previously realized (e.g., in the Arctic Biodiversity Assessment (Daniëls et al. 2013), only 190 non-natives were listed). Of these, 188 were naturalized in at least one of 23 Arctic regions, and 11 species were considered invasive. They additionally identified regions where the processes of non-native plant naturalization and invasion are particularly advanced (e.g., Alaska, Northern Iceland, and the Western part of the Russian Arctic). A comprehensive picture of important pathways by which non-native plant species are introduced to the Arctic emerged from the study, highlighting unintentional dispersal.

One of the most notable observations that emerged from assessing trends in vegetation FECs was the frequency with which change over time was either not significant or varied strongly by species or region. This aligns with many other studies that find high temporal and spatial variability in ongoing changes in the Arctic (e.g., Elmendorf et al. 2012; Guay et al. 2014; Myers-Smith et al. 2015; Vickers et al. 2016). For both abundance and phenology there is more often a change toward an increase or earlier start of the season in the southern and middle latitude regions of the Arctic. In the high Arctic, there are individual studies where change over time has been recorded, but less often than in the low Arctic. Bjorkman and others suggest one possible reason for such variability is that vegetation attributes are influenced by multiple drivers in each location, and the strength of the drivers likely varies between locations. Similarly, Jenkins et al. (2020) demonstrated that trends in remotely sensed phenology varied notably depending on the variable (e.g., green-up vs. senescence) and the bioclimatic subzone within which trends were evaluated. The many plant species and functional groups that constitute the composite vegetation variables identified for monitoring in CBMP have a wide range of life-history and evolutionary strategies and adaptations, and differing responses to environmental change. The Arctic as a habitat for plants also holds a great range of conditions, and the growing conditions at the local scale may vary almost as much as between large-scale regions due to small-scale heterogeneity within elevational, topographical, and biotic factors. Thus, the fact that there are very few uniform, panArctic trends in vegetation does not necessarily mean that Arctic vegetation is not changing, but rather that there are 
locally and regionally strong changes that depend on ecosystem and climate-specific conditions.

\section{Vegetation: Monitoring status}

The articles in this special issue compile monitoring data from remote sensing, plot-based studies, literature reviews, databases, and expert opinion. Nonetheless, many gaps remain, both in the data available and in our ability to interpret them. Jenkins et al. (2020) call for improved integration of data from multiple sources-harmonization, as described in the CBMP Arctic Terrestrial Biodiversity Monitoring Plan (Christensen et al. 2013)_for example, by validating satellite records and remote sensing data products with ground observations and modeling techniques. Integrating all three types of data would greatly improve our ability to detect trends and determine the spatial extent of these trends, as well as link these trends to particular drivers.

Bjorkman et al. (2020) highlight gaps in the kind of on-the-ground data collected and where it is collected; for example, long-term monitoring of phenology, particularly beginning and end-of-season phenology (e.g., leaf greenup and leaf senescence) is rare except in a handful of sites. In addition, there are relatively few Arctic locations where annual or near-annual vegetation monitoring is conducted; these include Svalbard (van der Wal and Stien 2014), the Norwegian mainland (Soininen et al. 2018), Greenland (Schmidt et al. 2017a), the Canadian high Arctic (Gauthier et al. 2011), and Alaska (Shaver and Jonasson 1999). There is a notable paucity of published monitoring records of any kind (phenology and composition/abundance) from much of the Siberian Arctic. The International Tundra Experiment (ITEX) (Henry and Molau 1997, https://www.gvsu.edu/itex/) and other networks such as the Shrub Hub (https://shrubhub.biology. ualberta.ca) contribute valuable information to long-term studies of plants and their responses to climate change. Within the networks, it varies greatly how stable and frequent the measurements are, varying from one re-visitation to annual monitoring.

In their review of invasive plant species in Arctic ecosystems, Wasowicz et al. (2020) note that current monitoring efforts are often not well suited to detect early stages of invasion. Instead, regular and well-planned monitoring of heavily disturbed and semi-natural plant communities should be carried out to detect taxa that are becoming naturalized as well as early stages of invasion, which would allow for more rapid action to mitigate the spread of invasive plants. In addition, the main entry points of non-native plant propagules should be identified and monitored on a regular basis.

\section{INVERTEBRATES}

In developing the Arctic Terrestrial Biodiversity Monitoring Plan (Christensen et al. 2013), the CBMP recognized that Arctic invertebrates play fundamental roles in northern ecosystems, arguably the most essential roles, and that they exhibit high diversity and abundance. Further, the plan states there is a fundamental need to understand changes in invertebrate populations and communities. To this end, it is vital to conduct long-term monitoring of invertebrates, "a topic that remains as critical today as when [others] discussed it 20 years ago" (Christensen et al. 2013, p. 49). This remains true today, and while some progress has been made, as has been presented in this issue, Arctic invertebrates remain grossly understudied and under-monitored. Given their inherent links to vegetation through herbivory, pollination, and soil nutrient cycling, and to mammals and birds through harassment, parasitism, and food provision (Fig. 1), there is enormous potential to rectify this through the CBMP, by monitoring invertebrates directly and through the combination of monitoring efforts across biomes and taxonomic groups.

\section{Invertebrates: Biodiversity status}

Gillespie et al. (2020a, b) review the status, at least to the extent possible, of Arctic arthropods. They conducted an assessment of biodiversity trends found in the literature, with a pan-Arctic focus on spiders, and a more detailed account of terrestrial arthropods in the North Atlantic region of the Arctic, "one of the best documented polar regions." Due to the paucity of baseline inventories and systematic monitoring, little can be said in detail about the status and trends of Arctic arthropods, even for such a relatively well-studied region. Gillespie et al. report that through a lack of ecological information, they often struggled to categorize species to the pre-determined FECs and subsequently to arrive at meaningful distribution patterns. However, they did produce a novel database on the occurrence patterns of 2519 arthropod species in faunistic regions of Greenland and the Islands of the North Atlantic, Iceland, Jan Mayen, and Svalbard. From this database, they offered broad inferences about diversity patterns and confirmed that arthropod diversity decreases with latitude, but that this generalization varies by arthropod group. Such conclusions do not merely corroborate previous findings and ecological logic (Danks 1981; Callaghan et al. 2004; Böcher et al. 2015); they highlight the pressing need for the initial stages of the CBMP to undertake basic investigations at each sampling station. For example, at each CBMP monitoring station, Gillespie et al. (2020a) strongly recommend the gathering of detailed local baseline information of species distribution, relative abundance, and relative 
importance to ecosystem services. Only then can monitoring be streamlined to quickly identify key indicator species, for example.

Despite the bleak outlook for describing the status of FECs in a well-known area, let alone in poorly studied or reported areas, Gillespie et al. (2020a, b) report on some indicative trends for limited, but promising, long-term datasets collected at Zackenberg and elsewhere in Greenland. These include the following:

- Significant declines in 7 of 14 muscid fly species were documented with $>80 \%$ decreases in diversity and abundance in some habitats between 1996 and 2014 (Loboda et al. 2018). Some of these species are important pollinators in some Arctic countries (Tiusanen et al. 2016), and their larvae may also perform important roles in freshwater systems (Michelsen 2015).

- A major group of decomposer invertebrates, Collembola, also showed declines in diversity in some habitats between 2007 and 2017 in Kobbefjord, West Greenland, but no changes in species richness over the same period. This may suggest increasing abundances of dominant species. Such a pattern is also found as species richness declines with latitude (Callaghan et al. 2004), but this suggestion requires corroboration.

- Variable trends in the species richness and total abundance of moths in Iceland, with significant declines in abundance at one sampling station, but increases in richness at two stations. However, the significance of trends depend on the timescale considered and varied widely, even between adjacent stations.

- Significant declines in abundance of certain spider species were also reported at Zackenberg for the period between 1996 and 2014. In particular, the decline in abundance of two habitat specialists is thought to be linked to climate warming and the earlier onset of spring (Bowden et al. 2018), and Gillespie et al. (2020b) suggest that climate-driven habitat changes may also be important.

- Overall abundances of invertebrate groups thought to form an important part of bird and mammal diets have also declined in Zackenberg between 1996 and 2016. As this trend was found by simply clustering many "important" families of invertebrate together, further work is required to determine the species or groups driving these trends and the likely causal factors, particularly if we seek to reverse or halt these worrying trends (Koltz et al. 2018).

The overall picture of trends in Arctic invertebrate diversity and abundance painted by Gillespie et al. $(2020 \mathrm{a}, \mathrm{b})$ is one of cautious concern. We do not have sufficient data from enough Arctic locations to set off alarm bells that ring as clearly as in other parts of the world (Hallmann et al. 2017; Lister and Garcia 2018), but indications from the few good datasets we do have mirror these temperate findings and may be like the prophetic canary in the coalmine. Gillespie et al. are reticent to say more due to the huge inherent spatial and temporal variability in Arctic invertebrate trends, but they urge further investigation to ensure we can make decisions that are more informed.

\section{Invertebrates: Monitoring status}

Core messages in describing the status of Arctic arthropod biodiversity in Gillespie et al. (2020a, b) include (1) the lack of available information, baseline inventories, and/or structured monitoring programs, to report on the biodiversity status, and (2) the spatial and temporal variability of trends suggest the need for comprehensive monitoring. Improving our basic knowledge of species inventories, ecology, and distribution will provide a stronger foundation for the CBMP, and ensuring monitoring in indicative habitats and across uninterrupted timescales will help researchers to distinguish natural variations from true species, diversity, and functional changes. For example, we may continue to find that the prey for vertebrates FECs is in decline in several Arctic areas, but without details about the key species, their ecology, the likely drivers of trends, and the spatial extent and variability of the phenomenon, we are unlikely to prescribe realistic remedial action. A further issue that arose from Gillespie et al. (2020b) in attempting to summarize the distribution and status of panArctic spider communities was that the information available is not organized according to same biogeographical boundaries (e.g., CAVM zones) and varies in quality between countries. This made it impossible to collate equivalent datasets for each country and Arctic zone in a timely manner and subsequently to draw conclusions and comparisons between regions. This highlights the need to standardize the recording and reporting aspect of biodiversity monitoring.

In summary, Gillespie et al. see enormous potential in the CBMP, because it sets out protocols for the gathering of widespread and long-term data that researchers of Arctic arthropods have been craving. This will build on on-going monitoring activities (Table 1 in Gillespie et al. 2020b) and the collaborative platform established by the Network for Arthropods of the Tundra (NeAT). However, this opportunity should also be planned carefully at each monitoring station, for example, by meeting the following:

- Baseline studies are needed at every station to locally uncover key species, habitats, ecological roles, and threats. 
- Abiotic data are required at relevant microhabitat scales.

- Funding should be secured to ensure continuous identification of the likely huge number of samples captured and to develop DNA libraries.

- Collaboration and communication between experts across regions and taxonomic specialisms are needed to ensure that monitoring opportunities are not missed. For example, invertebrates captured incidentally in sampling or studies of vegetation, soil, birds, and mammals could provide important insights that would be lost without cooperation throughout the CBMP.

\section{MAMMALS}

In the conceptual model of the CBMP Arctic Terrestrial Biodiversity Monitoring Plan (Christensen et al. 2013) (Fig. 1), mammals are the group with the lowest taxonomic diversity but include the largest of the Arctic terrestrial animals. They are fundamental to their ecosystems, often essential to the cultures and food security of Arctic peoples, and include charismatic species representing Arctic wilderness in people's imagination. The terrestrial monitoring plan identified six mammalian FECs: (i) large herbivores, (ii) medium sized herbivores/omnivores, (iii) small herbivores, (iv) large predators, (v) medium sized predators, and (vi) small predators. Of these six functional groups, muskoxen (Ovibos moschatus, Cuyler et al. 2020) and lemmings (Lemmus spp. and Dicrostonyx spp., Ehrich et al. 2020), representing large and small herbivores, respectively, and occupying key positions in tundra food webs, were addressed in this special issue.

Assessment of status and trend, associated with the CBMP terrestrial monitoring plan and the forthcoming State of the Arctic Terrestrial Biodiversity Report, but beyond the scope of this paper, is also available for two other taxa of Arctic mammals: wild reindeer/caribou (Rangifer tarandus, https://carma.caff.is/) and Arctic fox (Vulpes lagopus, Berteaux et al. 2017).

\section{Mammals: Biodiversity status}

Abundance is the FEC attribute for which most data exist for Arctic mammal populations. In the case of muskoxen, inferences regarding abundance across regions are complicated by differences in monitoring methodology and the large amount of variation in temporal and geographic extent of surveys. Population trajectories may also be the product of management actions. In addition to translocations, management may also involve harvest or removal of animals. Highlights of FEC attributes for muskoxen provided by Cuyler et al. (2020) include the following:

- Current circumpolar abundance estimate for muskoxen is higher than estimates from 2013 and 2017. Recent variations indicated that 23 muskox populations/regions were increasing, nine were stable, six were decreasing, and 17 were unknown. Of note, however, the two with steepest declines were, in 2000, the largest endemic populations in the world.

- Information on muskox demographics, health, and phenology is difficult to obtain and not widely collected or reported. This limits comparisons across populations.

- Although general distribution is well defined, detailed information on spatial structure is typically not available.

- Available genetic information indicates that muskox populations have gone through several bottlenecks and extirpation events resulting in low genetic variability, which created very low diversity in the major histocompatibility complex. Ability to respond to infectious diseases may be impaired. Further, translocated populations originated from relatively few animals, exacerbating already low diversity in some areas.

Lemmings are well known for their multi-annual density fluctuations, also known as lemming cycles, that create periodic pulses of herbivory pressure and resources for predators. Abundance data for lemmings showed large amounts of heterogeneity across years, sites, and species. Norwegian lemmings exhibited regular outbreaks at 3- to 6-year intervals, but sometimes much longer periods without outbreaks, and large variation in amplitude. Outside of Fennoscandia, heterogeneity was even greater. Regular cycles with a period of 3-4 years were observed at some sites, but this pattern varied considerably. In many cases, patterns were difficult to discern because of large differences in amplitude or monitoring methodology.

Due to the large variability in amplitude and regularity of lemming cycles, trends are inherently difficult to identify. Given that caveat, and the heterogeneity in existing data, as presented in Ehrich et al. (2020), available data indicate the following:

- No evidence that pan-Arctic lemming populations were increasing or decreasing over the last 25 years. Considering lemmings in different communities revealed a negative trend for low Arctic populations outside of Fennoscandia sympatric with voles. There were also indications of a negative trend in Russia, where several of the decreasing mixed community populations were located. 
- With one exception, all low Arctic lemming populations occurred in mixed small rodent communities including one or more species of voles.

- Change in species composition was noted at two low Arctic monitoring sites with meadow voles (Microtus pennsylvanicus) appearing in capture data in 2010 in Churchill Manitoba and $M$. middendorffii voles observed in South-eastern Taimyr in 2013 for the first time.

- For lemmings, health and phenology were seldom monitored. Demographic data were collected at about half of the 38 sites monitored but a synthesis of current trends is not available.

\section{Mammals: Monitoring status}

The two papers in this special issue reveal a considerable circumpolar effort to monitor Arctic mammal populations. Population size estimates were available for all 55 delineated muskox populations (Cuyler et al. 2020). Lemming population dynamics are currently monitored at 38 sites covering all biogeographic subzones and major geographic areas of the Arctic, mostly through a variety of abundance indices (Ehrich et al. 2020). For both FECs, it was difficult to assess overall trends because of large heterogeneity in methods and effort, infrequent surveys, and inconsistencies in the data in the case of muskoxen, and important geographic gaps in the case of lemming data. Unstandardized methods and variable data quality together with unrepresentative geographic coverage are likely to be the main challenges for monitoring trends in mammal populations in the Arctic today. Moreover, while abundance is assessed in all monitoring programs, demography, health, or spatial and genetic structure data (FEC attributes identified in the CBMP Arctic Terrestrial Biodiversity Monitoring Plan) are only rarely collected systematically.

Understanding trends resulting from the impact of ecological drivers is only possible if likely drivers are also measured at a relevant scale (Ims and Yoccoz 2017). Thus, both papers about mammals in this volume stress the necessity to apply a truly ecosystem-based approach to monitoring, in accordance with the recommendations of the CBMP. They highlight the lack of integration of important abiotic data into monitoring programs, such as the intensity and extent of adverse weather events or snow conditions at an appropriate scale. Large herbivores are an essential resource and integral to the cultures of Arctic peoples. Understanding the role of different drivers is crucial for appropriate management of animal populations in rapidly changing ecosystems and Arctic societies. Further, planning of ecosystem-based monitoring programs should include significant stakeholders, integrate local and traditional knowledge, and where possible communitybased monitoring (Cuyler et al. 2020).

Today's rapid and accelerating rates of change in the Arctic may exceed the adaptability and resilience of Arctic mammals. Relevant, timely monitoring, and accessible data are a priority. Past and present efforts are generally sporadic and poorly funded, regardless of species. While monitoring has increased in recent years, lack of standardization and data about drivers measured at appropriate scales remain major challenges for both analyses of specific population trends, but also circum-Arctic syntheses. Several CBMP mammal networks are currently engaged in finding solutions.

\section{BIRDS}

As outlined in the CBMP Arctic Terrestrial Biodiversity Monitoring Plan (Christensen et al. 2013, p. 75), "birds, as keystone consumers in Arctic ecosystems, are highly affected by the processes and drivers (biotic, abiotic and anthropogenic) that affect their food base...Furthermore, for many of the long-[distance] migrant species, the major factors affecting the abundance of avian species may operate outside of...the annual cycle when the birds are present in Arctic regions." The latter point makes birds unique because other FECs must cope with the Arctic winter, whereas the majority of Arctic-breeding bird species leave the Arctic in winter. Many of them move relatively short distances to temperate regions, while some winter in the tropics and the southern hemisphere. Thus, compared to other terrestrial FECs, birds are affected by a range of drivers over wider scales.

In this issue, circumpolar terrestrial bird FEC-attribute trends and monitoring coverage are discussed in three papers: Fuglei et al. (2020) focused on the Arctic-resident herbivores, rock ptarmigans (Lagopus muta), and willow ptarmigans (Lagopus lagopus); Franke et al. (2020) focused on top predators, the Arctic-resident gyrfalcon (Falco rusticolus), and the migrant peregrine falcon (Falco peregrinus); and Smith et al. (2020) reviewed the status of all main terrestrial bird populations ( 88 species included) according to functional groups (herbivore, insectivore, etc.), and 'flyways' (main migration routes) utilized by different populations.

\section{Birds: Biodiversity status}

Fuglei et al. (2020) compiled the first circumpolar synthesis of ptarmigan trends and added considerable broad-scale understanding of ptarmigan population dynamics. For rock ptarmigan, there was a significant, declining trend in density in 10 of 31 monitoring sites (time series), while a 
significant increase in density was found at five sites. For willow ptarmigan, there were significant negative density trends at two sites, and at three sites there were close to significant negative trends. Three sites showed significant positive trends. Multi-annual population cycles are common, but cycle period and amplitude varied spatially and temporally. Only 4 and 3 time series (monitoring sites) for rock ptarmigan and willow ptarmigan, respectively, failed to show evidence of cyclic dynamics. Both species displayed some periods with population cycles (3-6 year 'short,' and 9-12 year 'long' cycles), and cyclicity changed through time for some populations of both species. Long cycles appeared to be most prevalent in North America where they may be linked to snowshoe hare cycles (through an alternative prey mechanism, Boonstra et al. 2016) or in Iceland where they could be driven by gyrfalcon predation (gyrfalcon territory occupancy also cyclic, lagging after ptarmigan; Nielsen 2011). Short cycles were most prevalent in Scandinavia where they seem to be linked to rodent cycles. Interestingly, populations relatively close to each other, in some cases (e.g., NE and SE Iceland rock ptarmigans), had contrasting changes in cycle period over time-i.e., population peaks and lows became more frequent in some areas and less frequent in others nearby.

For the top avian predators, Franke et al. (2020) provided the first overview of monitoring sites for gyrfalcons and peregrine falcons. The authors analyzed long-term trends in occupancy and productivity and summarized information for recommended CBMP FEC attributes. At the circum-Arctic scale, for peregrine falcons, 9 of 12 monitoring sites indicated that occupancy was either stable or had increased over the course of monitoring, and three monitoring sites resulted in trends that indicated occupancy had declined. Seven of 10 peregrine falcon monitoring sites presented productivity trends that were either stable or increasing, and three resulted in trends that had declined. After experiencing lows in abundance in the 1970s, the peregrine falcon has recovered from pesticideinduced population crashes throughout the Nearctic and Palearctic. For gyrfalcons at the circumpolar scale, occupancy trends at 10 of 12 monitoring sites were found to be stable or had increased, whereas occupancy at the two remaining monitoring sites had declined. Productivity trends for gyrfalcons at 9 of 11 monitoring were stable, none showed evidence of increased productivity, and two presented trends that declined over the course of the monitoring period. For both species, monitored populations were considered to be generally stable, and assuming that these patterns hold beyond the temporal and spatial extents of the monitoring sites, it is reasonable to suggest that breeding populations at broader scales in the Arctic were similarly stable.
Taking a meta-view on terrestrial Arctic 'tundra birds' as FECs (88 species included), Smith et al.'s (2020) summary revealed that, overall, declines were most prevalent in waders (insectivores) and least prevalent in herbivore waterfowl (geese) whereas increasing population trends were most common in geese and least common in waders and other waterbirds. Within flyways, increases were generally most common in geese and least common among waders and waterbirds. Fewer waterfowl populations were increasing in the Central Asian and East Asian-Australasian flyways. The largest proportion of declining species was among the waders in all but the Central Asian flyways, where a large majority of waders had unknown trends.

Many species showed contrasting trends between different populations/flyways. This variation complicated our ability to draw broad conclusions, but findings to-date include the following:

- Most populations are faring well-65 species (74\%) had at least one increasing or stable flyway population.

- For more than half of all species, there are reasons for concern for some flyway populations: 50 species (57\%) had at least one population in decline, and for 22 species $(21 \%)$, all populations were declining.

- For $25 \%$ of the species, trends are unknown for at least one population; the largest proportion of unknown population trends are in the Central Asian flyway.

While several taxa declined, status is (so far) only known to be critical for a handful of species: the 'possibly extinct' (sensu IUCN Redlist terminology) Eskimo curlew (Numenius borealis), the 'critically endangered' Siberian crane (Leucogeranus leucogeranus) and spoon-billed sandpiper (Calidris pygmaea), three 'near threatened' subspecies of the red knot-the rufa in the Americas flyway and the pierssmai and rogersi in the East AsianAustralasian Flyway - and the 'near threatened' western Palearctic subpopulation of the lesser white-fronted goose (Smith et al. 2020).

\section{Birds: Monitoring status}

Ptarmigan populations have been monitored at over 90 sites, with about half of them still being monitored (Fuglei et al. 2020). Peregrine falcon and/or gyrfalcons are monitored at 24 sites (Franke et al. 2020). Waders and geese are monitored at many sites across the Arctic, but since these taxa are often gregarious at migration stopover and wintering sites, data on population trends are also supported by monitoring efforts outside the Arctic (CAFF 2018a). Data on passerines (mostly insectivores) and piscivores are scarcer. 
While the number of terrestrial bird-monitoring studies across the Arctic (Fig. 2) appears impressive, spatial coverage is uneven with most monitoring in the low Arctic (and sub-Arctic), Iceland, and Scandinavia, and relatively few in high Arctic; the Arctic Archipelago and Canada, Greenland, and central and eastern Russia are relatively poorly covered for all FECs.

Nevertheless, the current network of monitoring sites provides a good basis for further coordinated monitoring of bird populations and further analyses of trophic interactions and effects of external drivers. To further contribute to an ecosystem-based approach to monitoring, bird monitoring would need to address the following issues:

- Standardization of field protocols, data collection methods, terminology, and reporting to enhance data quality and facilitate comparisons among sites, including development of database tools for archiving data and semi-automated procedures capable of accumulating on-going data summaries.

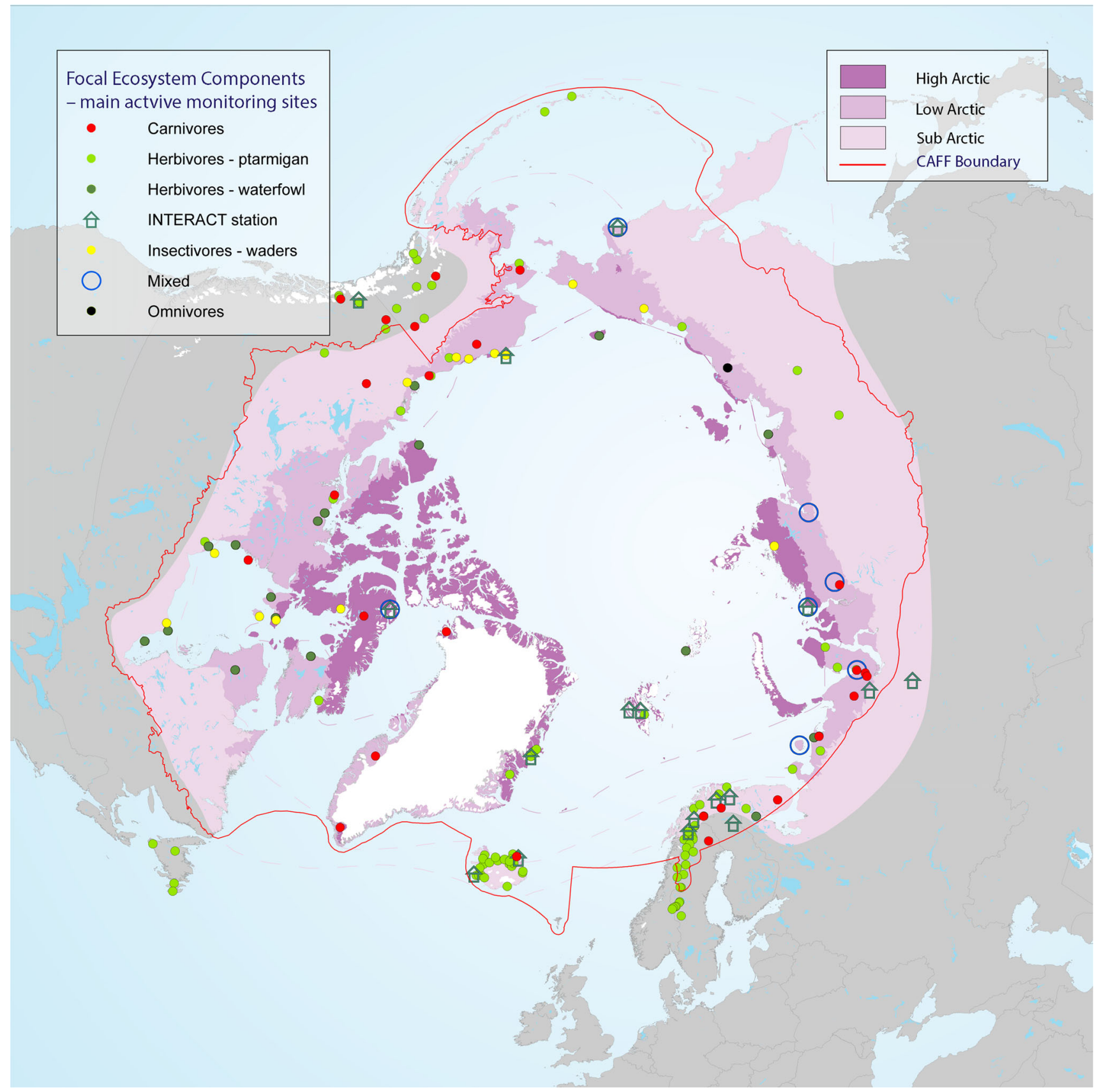

Fig. 2 Geographical coverage of terrestrial birds FEC monitoring in the Arctic; based on Arctic Shorebird Demographics Network (2014), INTERACT (2015), Fuglei et al. (2020), Franke et al. (2020), and M. Soloviev (pers. comm.) 
- Better integration within and among studies across trophic levels. For example, gyrfalcon occupancy (Falkdalen et al. 2011; Nielsen 2011) and reproductive success (Barichello and Mossop 2011; Koskimies 2011) are known to depend on ptarmigan cycles.

- Better integration of factors that underpin changes in phenology, demography, and abundance.

- Collection of non-invasive samples for analyses of genetics, isotopes, and contaminants (e.g., molted feathers, pellets, eggshells, addled eggs, prey remains).

- Promotion of community-based monitoring, use of citizen science, and application of traditional knowledge.

- Promotion of studies engaged in collecting multispecies, and long-term time series data for estimating trophic interactions, particularly those associated with cyclic patterns. For example, ptarmigan and gyrfalcons, and small mammals and peregrine falcons, gyrfalcons, snowy owls (Bubo scandiacus), rough-legged buzzards (Buteo lagopus), and jaegers (Stercorarius spp).

\section{DISCUSSION}

That the Arctic is changing is beyond doubt, as is illustrated by the papers presented in this special issue. While numerous characteristics of the Arctic are exhibiting directional change (Box et al. 2019), the papers assembled here highlight heterogeneity as a predominant pattern particularly when assessing global trends of change for Arctic terrestrial biodiversity. This was the case, at least to some extent, for each of the FECs in the CBMP terrestrial conceptual model (Fig. 1). Geographic variability in trends and species-specific responses to environmental changes were consistent patterns revealed for vegetation, spatial and temporal variability in documented trends for invertebrates was predominant, and similar, high variation was reported for FEC attributes for birds and mammals. Bjorkman et al. (2020) suggest that vegetation attributes are influenced by multiple drivers in each location, and that the strength of the drivers varies by location, a contention which is likely to be valid for other FECs as well. It is therefore pivotal, but also a key challenge for CBMP as a pan-Arctic monitoring program, to conduct monitoring at all relevant scales, temporally as well as spatially.

The documented variability highlights the difficulty in synthesizing results at a pan-Arctic scale and necessitates eco-regionally specific conceptual models to guide biodiversity monitoring locally and regionally. Such conceptual models would help to ensure focus on the most likely drivers of biodiversity change in each region and ensure that monitoring protocols include necessary variables to understand causal links between drivers and changes in FEC attributes. Further, averaging observations over many localities and thus over many different ecoregions logically results in the absence of statistically significant overall trends of change, as regional variations are smoothed. This is counterproductive to understanding Arctic biodiversity change. Rather than searching unsuccessfully for circumpolar trends, we should, perhaps, instead ask how, where, and why there is variation in the response of Arctic FECs to environmental changes.

The Arctic is home to a diverse and unique set of species. Being species-poor compared to lower latitudes, Arctic food webs have been regarded as being rather simple (Legagneux et al. 2012). Recent studies, however, have described Arctic webs in unprecedented detail and have exposed complex and highly interconnected interactions (Wirta et al. 2014; Schmidt et al. 2017a). This connectedness has implications for how the system responds to environmental changes, as impacts on one trophic level may propagate onto other levels. For instance, recent reports of increased temporal mismatch between plant flowering and their pollinators, induced by warmer climates, may have repercussions for plants and arthropods (Høye et al. 2013; Schmidt et al. 2016), but the negative impacts of warming may even cascade onto another trophic level-insectivorous birds (Reneerkens et al. 2016).

To more fully understand the impacts of environmental change requires a long-term perspective and evaluation across multiple scales and interactions, simultaneously. The CBMP is predicated on an ecosystem approach and conceptual models that identify important linkages and cross-biome interactions. Although the papers in this issue often allude to the importance of these interactions, most have had to rely on monitoring and/or research based on a reductionist approach. The exception is Jenkins et al. (2020), in which the authors analyze terrestrial and marine FECs and potential drivers simultaneously, across space and time, in part, looking for cross-biome relationships and commonalities in trend, if only correlative in nature. We strongly urge the CBMP to advance Objective 3.3: Develop integrated and targeted State of the Arctic Biodiversity Reporting, in the "Circumpolar Biodiversity Monitoring Program Strategic Plan: 2018-2021" (CAFF 2018b). In achieving this objective, the CBMP could accomplish the desired "ecosystem-based program of work that considers biodiversity elements, system drivers, and interactions within and across the marine, coastal, freshwater, and terrestrial environments,... [simultaneously, and] achieve a single, circumpolar, interdisciplinary State of the Arctic Biodiversity Report..."

An extensive, integrated, ecosystem-based monitoring program would preferably be coupled with targeted research projects deciphering processes causing patterns 
observed in monitoring programs (Schmidt et al. 2017b). The CBMP Arctic Terrestrial Biodiversity Monitoring Plan recommends a balance of targeted, research-based monitoring (e.g., Lindenmayer and Lichens 2010) and surveybased status and trend monitoring in order to increase the geographic extent over which changes can be detected (Christensen et al. 2013). While survey-based monitoring contributes to understanding how biodiversity elements are changing across time and space, the papers in this special issue clearly illustrate limitations of this approach alone. Large-scale surveys based on existing data gathered for multiple purposes (well exemplified by the small rodent monitoring data; Ehrich et al. 2020) do not allow causal mechanisms to be identified. We recognize that, as opposed to physical attributes of the environment, predicting the ecological responses to future climate, for instance, is prone to much larger uncertainties (Urban 2019). Nonetheless, developing an improved mechanistic understanding of how Arctic ecosystems work, and coupling this with systematic long-term monitoring of status and trends, would allow us to track both changes in Arctic FECs (biodiversity) and the likely drivers of that change. We would, then, be able to provide knowledge targeted toward informing national resource management decision-making and national and international conservation initiatives and commitments.

Acknowledgements The CBMP, toward program implementation and development of the State of the Arctic Terrestrial Biodiversity Report, has received financial and capacity support from the following sources: Denmark/Greenland, the Danish Environmental Protection Agency as part of the Danish environmental support programme Dancea, Canada, Iceland, Sweden, United States, and the Nordic Council of Ministers. Also, the authors acknowledge the contributions of members of the CBMP Terrestrial Steering Group.

\section{REFERENCES}

Arctic Shorebird Demographics Network. 2014. Breeding Camp Protocol Version 5, April 2014. https://www.manomet.org/wpcontent/uploads/old-files/ASDN_Protocol_V5_20Apr2014.pdf.

Barichello, N., and D. Mossop. 2011. The overwhelming influence of Ptarmigan abundance on Gyrfalcon reproductive success in the central Yukon, Canadan. In Gyrfalcons and ptarmigan in a changing world, vol. I, ed. R.T. Watson, T.J. Cade, M. Fuller, G. Hunt, and E. Potapov, 307-322. Boise: The Peregrine Fund.

Beever, E., and A. Woodward. 2011. Design of ecoregional monitoring in conservation areas of high-latitude ecosystems under contemporary climate change. Biological Conservation 144: 1258-1269. https://doi.org/10.1016/j.biocon.2010.06.022.

Berteaux, D., A.-M. Thierry, R. Alisauskas, A. Angerbjörn, E. Buchel, L. Doronina, D. Ehrich, N. Eide, et al. 2017. Harmonizing circumpolar monitoring of Arctic fox: Benefits, opportunities, challenges and recommendations. Polar Research. https:// doi.org/10.1080/17518369.2017.1319602.

Bjorkman, A.D., M.G. Criado, I.H. Myers-Smith, V. Ravolainen, I.S. Jónsdóttir, K.B. Westergaard, J.P. Lawler, M. Aronsson, et al.
2020. Status and trends in Arctic vegetation: Evidence from experimental warming and long-term monitoring. In Terrestrial biodiversity in a rapidly changing Arctic, eds. N.M. Schmidt, and H. Jóhannesdóttir, Ambio vol. 49, Special Issue. https://doi. org/10.1007/s13280-019-01161-6.

Böcher, J., N.P. Kristensen, T. Pape, and L. Vilhelmsen (eds.). 2015. The greenland entomofauna: An identification manual of insects, spiders and their allies. Leiden: Koninklijke Brill nv.

Boonstra, R., H.P. Andreassen, S. Boutin, J. Husek, R.A. Ims, C.J. Krebs, C. Skarpe, and P. Wabakken. 2016. Why do the boreal forest ecosystems of northwestern Europe differ from those of western North America? BioScience 66: 722-734. https://doi. org/10.1093/biosci/biw080.

Box, J.E., W.T. Colgan, T.R. Christensen, N.M. Schmidt, M. Lund, F.J. Parmentier, R. Brown, U.S. Bhatt, et al. 2019. Key indicators of Arctic climate change: 1971-2017. Environmental Research Letters 14: 045010. https://doi.org/10.1088/1748-9326/aafc1b.

Bowden, J.J., O.L.P. Hansen, K. Olsen, N.M. Schmidt, and T.T. Høye. 2018. Drivers of inter-annual variation and long-term change in high-Arctic spider species abundances. Polar Biology 41: 1635-1649. https://doi.org/10.1007/s00300-018-2351-0.

CAFF. 2018a. A global audit of the status and trends of Arctic and Northern Hemisphere goose populations (Component 2: Population accounts). Conservation of Arctic Flora and Fauna International Secretariat, Akureyri, Iceland. ISBN 978-9935431-74-5.

CAFF. 2018b. Circumpolar biodiversity monitoring program strategic plan 2018-2021. CAFF monitoring series report no. 29. Conservation of Arctic flora and fauna, Akureyri, Iceland. ISBN: 978-9935-431-71-4.

Callaghan, T.V., L.O. Björn, Y. Chernov, T. Chapin, T.R. Christensen, B. Huntley, R.A. Ims, M. Johansson, et al. 2004. Biodiversity, distributions and adaptations of arctic species in the context of environmental change. Ambio 33: 404-417. https:// doi.org/10.1579/0044-7447-33.7.404.

Christensen, T., J. Payne, M. Doyle, G. Ibarguchi, J. Taylor, N.M. Schmidt, M. Gill, M. Svoboda, et al. 2013. The Arctic terrestrial biodiversity monitoring plan. CAFF monitoring series report no. 7. CAFF International Secretariat, Akureyri, Iceland. https://caff. is/publications/view_document/256-arctic-terrestrialbiodiversity-monitoring-plan. ISBN 1.978-9935-431-26-4.

Culp, J.M., W. Goedkoop, J. Lento, K.S. Christoffersen, S. Frenzel, G. Guðbergsson, P. Liljaniemi, S. Sandøy, et al. 2012. The Arctic freshwater biodiversity monitoring plan. CAFF International Secretariat, CAFF monitoring series report no. 7. CAFF International Secretariat. Akureyri, Iceland. https://caff.is/ freshwater/freshwater-monitoring-publications/196-arctic-

freshwater-biodiversity-monitoring-plan. ISBN 978-9935-43119-6.

Cuyler, C., J. Rowell, J. Adamczewski, M. Anderson, J. Blake, T. Bretten, V. Brodeur, M. Campbell, et al. 2020. Muskox status, recent variation, and uncertain future. In Terrestrial biodiversity in a rapidly changing Arctic, eds. N.M. Schmidt, and H. Jóhannesdóttir, Ambio vol. 49, Special Issue. https://doi.org/10. 1007/s13280-019-01205-x.

Daniëls, F.J.A, L.J. Gillespie, and M. Poulin. 2013. Plants. In: Arctic biodiversity assessment. Status and trends in Arctic biodiversity, eds. H. Meltofte, A.B. Josefson, and D. Payer, pp. 259-301. Akureyri: CAFF. http://arcticlcc.org/assets/resources/ ABA2013Science.pdf.

Danks, H.V. 1981. Arctic Arthropods. A review of systematics and ecology with particular reference to the North American fauna. Entomological Society of Canada, Ottawa.

Ehrich, D., N.M. Schmidt, G. Gauthier, R. Alisauskas, A. Angerbjörn, K. Clark, F. Ecke, N.E. Eide, et al. 2020. Documenting lemming population change in the Arctic: Can we detect trends? In 
Terrestrial biodiversity in a rapidly changing Arctic, eds. N.M. Schmidt, and H. Jóhannesdóttir, Ambio vol. 49, Special Issue. https://doi.org/10.1007/s13280-019-01198-7.

Elmendorf, S.C., G.H.R. Henry, R.D. Hollister, R.G. Bjork, A.D. Bjorkman, T.V. Callaghan, L.S. Collier, E.J. Cooper, et al. 2012. Global assessment of experimental climate warming on tundra vegetation: Heterogeneity over space and time. Ecology Letters 15: 164-175. https://doi.org/10.1111/j.1461-0248.2011.01716.x.

Falkdalen, U., M. Hörnell-Willebrand, T. Nygård, T. Bergström, G. Lind, A. Nordin, and B. Warensjö. 2011. Relations between willow ptarmigan (Lagopus lagopus) density and gyrfalcon (Falco rusticolus) breeding performance in Sweden. In Gyrfalcons and ptarmigan in a changing world, vol. I, ed. R.T. Watson, T.J. Cade, M. Fuller, G. Hunt, and E. Potapov, 171-176. Boise: The Peregrine Fund.

Franke, A., K. Falk, K. Hawkshaw, S. Ambrose, D.L. Anderson, P.J. Bente, T. Booms, K.K. Burnham, et al. 2020. Status and trends of circumpolar peregrine falcon and gyrfalcon populations. In Terrestrial biodiversity in a rapidly changing Arctic, eds. N.M. Schmidt, and H. Jóhannesdóttir, Ambio vol. 49, Special Issue. https://doi.org/10.1007/s13280-019-01300-z.

Fuglei, E., J.-A. Henden, C.T. Callahan, O. Gilg, J. Hansen, R.A. Ims, A.P. Isaev, J. Lang, et al. 2020. Circumpolar status of Arctic ptarmigan: Population dynamics and trends. In Terrestrial biodiversity in a rapidly changing Arctic, eds. N.M. Schmidt, and H. Jóhannesdóttir, Ambio vol. 49, Special Issue. https://doi. org/10.1007/s13280-019-01191-0.

Gauthier, G., D. Berteaux, J. Bêty, A. Tarroux, J.-F. Therrien, L. McKinnon, P. Legagneux, and M.-C. Cadieux. 2011. The tundra food web of Bylot Island in a changing climate and the role of exchanges between ecosystems. Ecoscience 18: 223-235. https:// doi.org/10.2980/18-3-3453.

Gilg, O., K.M. Kovacs, J. Aars, J. Fort, G. Gauthier, D. Gremillet, R.A. Ims, H. Meltofte, et al. 2012. Climate change and the ecology and evolution of Arctic vertebrates. Annals of the New York Academy of Science 1249: 166-190. https://doi.org/10. 1111/j.1749-6632.2011.06412.x.

Gillespie, M.A.K., M. Alfredsson, I.C. Barrio, J.J. Bowden, P. Convey, L.E. Culler, S.J. Coulson, P.H. Krogh, et al. 2020a. Status and trends of terrestrial arthropod abundance and diversity in the North Atlantic region of the Arctic. In Terrestrial biodiversity in a rapidly changing Arctic, eds. N.M. Schmidt, and H. Jóhannesdóttir, Ambio vol. 49, Special Issue. https://doi. org/10.1007/s13280-019-01162-5.

Gillespie, M.A.K., M. Alfredsson, I.C. Barrio, J. Bowden, P. Convey, S.J. Coulson, L.E. Culler, M.T. Dahl, et al. 2020b. Circumpolar terrestrial arthropod monitoring: A review of ongoing activities, opportunities and challenges, with a focus on spiders. In Terrestrial biodiversity in a rapidly changing Arctic, eds. N.M. Schmidt, and H. Jóhannesdóttir, Ambio vol. 49, Special Issue. https://doi.org/10.1007/s13280-019-01185-y.

Gill, M.J., K. Crane, R. Hindrum, P. Arneberg, I. Bysveen, N.V. Denisenko, V. Gofman, and A. Grant-Friedman. 2011. Arctic marine biodiversity monitoring plan. CAFF monitoring series report no. 3. CAFF International Secretariat, Akureyri, Iceland. https://caff.is/monitoring-series/view_document/3-arctic-marinebiodiversity-monitoring-plan. ISBN 1.978-9979-9778-7-2.

Guay, K.C., P.S.A. Beck, L.T. Berner, S.J. Goetz, A. Baccini, and W. Buermann. 2014. Vegetation productivity patterns at high northern latitudes: A multi-sensor satellite data assessment. Global Change Biology 20: 3147-3158. https://doi.org/10.1111/ gcb.12647.

Hallmann, C.A., M. Sorg, E. Jongejans, H. Siepel, N. Hofland, H. Schwan, W. Stenmans, A. Müller, et al. 2017. More than 75 percent decline over 27 years in total flying insect biomass in protected areas. PLOS ONE 12: e0185809. https://doi.org/10. 1371/journal.pone.0185809.

Henry, G., and U. Molau. 1997. Tundra plants and climate change: The International Tundra Experiment (ITEX). Global Change Biology 3: 1-9. https://doi.org/10.1111/j.1365-2486.1997. gcb132.x.

Høye, T.T., E. Post, N.M. Schmidt, K. Trøjelsgaard, and M.C. Forchhammer. 2013. Shorter flowering seasons and declining abundance of flower visitors in a warmer Arctic. Nature Climate Change 3: 759-763. https://doi.org/10.1038/nclimate1909.

Ims, R.A., and N.G. Yoccoz. 2017. Ecosystem-based monitoring in the age of rapid climate change and new technologies. Current Opinion in Environmental Sustainability 29: 170-176. https:// doi.org/10.1016/j.cosust.2018.01.003.

INTERACT. 2015. INTERACT Research and Monitoring, eds E. Topp-Jørgensen., Z. Tairova, M. Rasch, and J. Hansen. DCE Danish Centre for Environment and Energy, Aarhus University, Denmark.

IPCC. 2019. IPCC Special Report on the Ocean and Cryosphere in a Changing Climate, eds. H.-O. Pörtner, D.C. Roberts, V. MassonDelmotte, P. Zhai, M. Tignor, E. Poloczanska, K. Mintenbeck, M. Nicolai, et al. https://www.ipcc.ch/srocc/download-report/.

Jenkins, L.K., T. Barry, K.R. Bosse, W.S. Currie, T. Christensen, S. Longan, R.A. Shuchman, D. Tanzer, et al. 2020. Satellite-based decadal change assessments of pan-Arctic environments. In Terrestrial biodiversity in a rapidly changing Arctic, eds. N.M. Schmidt, and H. Jóhannesdóttir, Ambio vol. 49, Special Issue. https://doi.org/10.1007/s13280-019-01249-z.

Jones, T., D. McLennan, C. Behe, M. Arvnes, S. Wesseberg, L. Sergienko, C. Harris, Q. Harcharek, and T. Christensen. 2019. Arctic coastal biodiversity monitoring plan. Conservation of Arctic Flora and Fauna International Secretariat: Akureyri, Iceland. https://caff.is/coastal/coastal-monitoring-publications/ 473-arctic-coastal-biodiversity-monitoring-plan. 978-9935-431-76-9.

Koltz, A.M., N.M. Schmidt, and T.T. Høye. 2018. Differential arthropod responses to warming are altering the structure of Arctic communities. Royal Society Open Science. https://doi.org/ 10.1098/rsos.171503.

Koskimies, P. 2011. Conservation biology of the gyrfalcon (Falco rusticolus) in northern Fennoscandia. In Gyrfalcons and ptarmigan in a changing world, ed. R.T. Watson, T.J. Cade, M. Fuller, G. Hunt, and E. Potapov, 95-124. Boise: The Peregrine Fund.

Legagneux, P., G. Gauthier, D. Berteaux, J. Bêty, M.C. Cadieux, F. Bilodeau, E. Bolduc, L. McKinnon, et al. 2012. Disentangling trophic relationships in a High Arctic tundra ecosystem through food web modeling. Ecology 93: 1707-1716. https://doi.org/10. 1890/11-1973.1.

Lindenmayer, D.B., and G.E. Lichens. 2010. The science and application of ecological monitoring. Biological Conservation 143: 1317-1328. https://doi.org/10.1016/j.biocon.2010.02.013.

Lister, B.C., and A. Garcia. 2018. Climate-driven declines in arthropod abundance restructure a rainforest food web. Proceedings of the National Academy of Sciences 115: E10397E10406. https://doi.org/10.1073/pnas.1722477115.

Loboda, S., J. Savage, C.M. Buddle, N.M. Schmidt, and T.T. Høye. 2018. Declining diversity and abundance of High Arctic fly assemblages over two decades of rapid climate warming. Ecography 41: 265-277. https://doi.org/10.1111/ecog.02747.

Meltofte, H. (ed.). 2013. Arctic biodiversity assessment. Status and trends in Arctic biodiversity. Akureyri: Conservation of Arctic Flora and Fauna.

Michelsen, V. 2015. Anthomyiidae, Fannidae, Muscidae and Scathophagidae (the Muscidae family group). In The Greenland Entomofauna: An identification manual of insects, spiders and 
their allies, ed. J. Böcher, K. Kristensen, T. Pape, and L. Vilhelmsen. Leiden: Koninklijke Brill.

Myers-Smith, I.H., S.C. Elmendorf, P.S.A. Beck, M. Wilmking, M. Hallinger, D. Blok, K.D. Tape, S.A. Rayback, et al. 2015. Climate sensitivity of shrub growth across the tundra biome. Nature Climate Change 5: 887-891. https://doi.org/10.1038/ nclimate2697.

Nielsen, O.K. 2011. Gyrfalcon population and reproduction in relation to rock ptarmigan numbers in Iceland. In Gyrfalcons and ptarmigan in a changing world, vol. II, ed. R.T. Watson, T.J. Cade, M. Fuller, G. Hunt, and E. Potapov, 21-48. Boise: The Peregrine Fund.

Ravolainen, V., E.M. Soininen, I.S. Jónsdóttir, I. Eischeid, M. Forchhammer, R. van der Wal, and Å. . Pedersen. 2020. High Arctic ecosystem states: Conceptual models of vegetation change to guide long-term monitoring and research. In Terrestrial biodiversity in a rapidly changing Arctic, eds. N.M. Schmidt, and H. Jóhannesdóttir, Ambio vol. 49, Special Issue. https://doi.org/10.1007/s13280-019-01310-x.

Reneerkens, J., N.M. Schmidt, O. Gilg, J. Hansen, L.H. Hansen, J. Moreau, and T. Piersma. 2016. Effects of food abundance and early clutch predation on reproductive timing in high Arctic shorebirds exposed to advancements in arthropod abundance. Ecology and Evolution 6: 7375-7386. https://doi.org/10.1002/ ece3.2361

Schmidt, N.M., B. Hardwick, O. Gilg, T.T. Høye, P.H. Krogh, H. Meltofte, A. Michelsen, J.B. Mosbacher, et al. 2017a. Interaction webs in arctic ecosystems: Determinants of arctic change? Ambio 46: 12-25. https://doi.org/10.1007/s13280-016-0862-x.

Schmidt, N.M., J.B. Mosbacher, P.S. Nielsen, C. Rasmussen, T.T. Høye, and T. Roslin. 2016. An ecological function in crisis? The temporal overlap between plant flowering and pollinator function shrinks as the Arctic warms. Ecography 39: 1250-1252. https:// doi.org/10.14430/arctic4656.

Schmidt, N.M., T.R. Christensen, and T. Roslin. 2017b. A high arctic experience of uniting research and monitoring. Earth's Future 5: 650-654. https://doi.org/10.1002/2017EF000553.

Shaver, G., and S. Jonasson. 1999. Response of Arctic ecosystems to climate change: Results of long-term field experiments in Sweden and Alaska. Polar Research 18: 245-252. https://doi. org/10.1111/j.1751-8369.1999.tb00300.x.

Smith, P.A., L. McKinnon, H. Meltofte, R.B. Lanctot, A.D. Fox, J.O. Leafloor, M. Soloviev, A. Franke, et al. 2020. Status and trends of tundra birds across the circumpolar Arctic. In Terrestrial biodiversity in a rapidly changing Arctic, eds. N.M. Schmidt, and H. Jóhannesdóttir, Ambio vol. 49, Special Issue. https://doi. org/10.1007/s13280-019-01308-5.

Soininen, E.M., J.-A. Henden, V.T. Ravolainen, N.G. Yoccoz, K.A. Bråthen, S.T. Killengreen, and R.A. Ims. 2018. Transferability of biotic interactions: Temporal consistency of arctic plant-rodent relationships is poor. Ecology and Evolution 8: 9697-9711. https://doi.org/10.1002/ece3.4399.

Tiusanen, M., P.D.N. Hebert, N.M. Schmidt, and T. Roslin. 2016. One fly to rule them all-muscid flies are the key pollinators in the Arctic. Proceedings of the Royal Society B-Biological Sciences 283: 8. https://doi.org/10.1098/rspb.2016.1271.

Urban, M.C. 2019. Projecting biological impacts from climate change like a climate scientist. WIREs Climate Change. https://doi.org/ $10.1002 /$ wcc. 585

Vickers, H., K.A. Hogda, S. Solbo, S.R. Karlsen, H. Tommervik, R. Aanes, and B.B. Hansen. 2016. Changes in greening in the high Arctic: Insights from a 30 year AVHRR max NDVI dataset for Svalbard. Environmental Research Letters 11: 105004. https:// doi.org/10.1088/1748-9326/11/10/105004. van der Wal, R., and A. Stien. 2014. High-arctic plants like it hot: A long-term investigation of between-year variability in plant biomass. Ecology 95: 3414-3427. https://doi.org/10.1890/140533.1.

Wasowicz, P., A.N. Sennikov, K.B. Westergaard, K. Spellman, M. Carlson, L.J. Gillespie, J.M. Saarela, S.S. Seefeldt, et al. 2020. Non-native vascular flora of the Arctic: Taxonomic richness, distribution and pathways. In Terrestrial biodiversity in a rapidly changing Arctic, eds. N.M. Schmidt, and H. Jóhannesdóttir, Ambio vol. 49, Special Issue. https://doi.org/10.1007/s13280019-01296-6.

Wirta, H.K., P.D. Hebert, R. Kaartinen, S.W. Prosser, G. Várkonyi, and T. Roslin. 2014. Complementary molecular information changes our perception of food web structure. Proceedings of the National Academy of Sciences 111: 1885-1890. https://doi.org/ 10.1073/pnas.1316990111.

Publisher's Note Springer Nature remains neutral with regard to jurisdictional claims in published maps and institutional affiliations.

\section{AUTHOR BIOGRAPHIES}

Jason J. Taylor $(\bowtie)$ Ph.D. is a protected areas Superintendent for the US National Park Service, in Alaska. He is a past Co-Lead for the Circumpolar Biodiversity Monitoring Program and served as the US Representative to the CBMP Terrestrial Steering Group for many years. His research efforts have focused on landscape ecology and multi-scale monitoring programs.

Address: U.S. National Park Service, PO Box 517, Skagway, AK 99840, USA.

e-mail: Jason_J_Taylor@nps.gov

James P. Lawler Ph.D. is the Program Manager for an Ecological Inventory and Monitoring program for the US National Park Service, in Alaska. James serves on the CBMP Terrestrial Steering Group as a US Representative. James's research interests are primarily large mammal ecology and monitoring of northern environments.

Address: U.S. National Park Service, 240 West 5th Ave, Anchorage, AK 99501, USA.

e-mail: jim_lawler@nps.gov

Mora Aronsson is a Senior Advisor at Swedish Species Information Centre, Swedish University of Agricultural Sciences, and Co-Chair for Terrestrial CBMP over the last 4 years. He has for 40 years been working with many aspects of biodiversity in Sweden, the European Union, and the Arctic, and has a special interest in Arctic/sub-Arctic vascular plants and vegetation.

Address: Swedish Species Information Centre, Swedish University of Agricultural Sciences, P.O. Box 7007, 75007 Uppsala, Sweden. e-mail: mora.aronsson@slu.se

Tom Barry is the Executive Secretary for the Conservation of Arctic Flora and Fauna (CAFF), the biodiversity working group of the Arctic Council, based in Akureyri, Iceland. Tom works with a diverse range of stakeholders from across the Arctic to ensure that biodiversity conservation remains a priority for audiences working in Arctic policy and in multilateral global environmental agreement settings. Address: CAFF Secretariat Borgir, Nordurslod 600, Akureyri, Iceland. Address: Department of the Environment and Natural Resources, University of Iceland, Sæmundargata 2, 102 Reykjavík, Iceland. e-mail: tom@caff.is 
Anne D. Bjorkman is a Senior Lecturer in Plant Ecology at the University of Gothenburg. Her research interests include biogeography, plant functional ecology, and community ecology, with a particular focus on the ecological consequences of climatic change in tundra ecosystems.

Address: Department of Biological and Environmental Sciences, University of Gothenburg, Carl Skottsbergs gata 22B, 40530 Göteborg, Sweden.

e-mail: annebj@gmail.com

Tom Christensen is a Co-Lead of the Circumpolar Biodiversity Monitoring Program and Section Leader, Section of Arctic Environment, Aarhus University. His main research areas include development of methods to identify important biodiversity areas and environmental consequences of human impacts on biodiversity hot spots in the Arctic. He is also experienced in the development of monitoring standards for Arctic biodiversity and data harmonization and integration.

Address: Arctic Research Centre, Department of Bioscience, Aarhus University, Frederiksborgvej 399, 4000 Roskilde, Denmark.

e-mail: toch@bios.au.dk

Stephen J. Coulson Ph.D. is a member of the Terrestrial Expert Monitoring Group within the CBMP and author for publications in the present special issue. He has been active in Arctic science since 1991 with a primary focus on the invertebrate biology of terrestrial and freshwater habitats in Svalbard.

Address: Swedish Species Information Centre, Swedish University of Agricultural Sciences, P.O. Box 7007, 75007 Uppsala, Sweden.

e-mail: stephen.coulson@slu.se

Christine Cuyler Ph. D. is a Senior Scientist at PinngortitaleriffikGreenland Institute of Natural Resources, working with caribou and muskoxen stock assessment, demographics, distribution and habitat use, health, drivers of change, threats, and methods development. The primary purpose is providing knowledge relevant to recommendations underlying management for sustainable use.

Address: Greenland Institute of Natural Resources, P.O. Box 570, 3900 Nuuk, Greenland.

e-mail: chris.cuyler@natur.gl

Dorothee Ehrich is a Researcher at UiT-The Arctic University of Norway, working in the Climate Ecological Observatory for Arctic Tundra. Her research focuses on changes in tundra ecosystems related to climate and human activities, in particular trophic interactions.

Address: UiT The Arctic University of Norway, Framstredet 39, 9037 Tromsø, Norway.

e-mail: dorothee.ehrich@uit.no

Knud Falk is an Independent Researcher and Consultant on Arctic biodiversity monitoring and management, since 1981, together with Søren Møller, leading peregrine falcon monitoring in South Greenland.

Address: Stockholm, Sweden.

e-mail: knudfalk@hotmail.com

Alastair Franke is a Principal Investigator of the Arctic Raptors Project; a research group working in Nunavut, Canada, focused on studying the ecology of Arctic-nesting raptors and their prey within the context of climate change. He holds an adjunct professorship in the Department of Biological Sciences at the University of Alberta, Edmonton, Alberta, Canada.

Address: Department of Biological Sciences, University of Alberta, Biological Sciences Bldg., CW 405, Edmonton, AB T6G 2E9, UK.
Address: Arctic Raptor Project, P.O. Box 626, Rankin Inlet, NT X0C 0G0, Canada.

e-mail: alastair.franke@ulaberta.ca

Eva Fuglei is a Senior Researcher at the Norwegian Polar Institute, Norway. Her research interests are within high arctic tundra ecosystems, climate change and human impacts, terrestrial animal ecology, management of arctic ecosystem services and biodiversity, and adaptive monitoring.

Address: Norwegian Polar Institute, Fram Centre, Postbox 6606, Langnes 9296, Troms $\varnothing$, Norway.

e-mail: eva.fuglei@npolar.no

Mark A. Gillespie is an Associate Professor at the Western Norway University of Applied Sciences. His research interests include insect ecology and plant-insect interactions.

Address: Department of Engineering and Natural Science, Western Norway University of Applied Sciences, Sogndal Campus, 6851 Sogndal, Norway.

e-mail: markg@hvl.no

Starri Heiðmarsson is a Botanist at the Icelandic Institute of Natural History, curator of the lichen herbarium, AMNH, and Co-Chair of Terrestrial CBMP over the last 3 years. His research interests are lichen biodiversity of Iceland and the Arctic, primary succession on nunataks, and the taxonomy of the primarily lichenized family Verrucariaceae.

Address: Icelandic Institute of Natural History, Borgir Nordurslod, 600 Akureyri, Iceland.

e-mail: starri@ni.is

Toke Høye is a Senior Researcher at the Aarhus University. His research interests include the effects of global change on populations of plants and animals. He leads the Network for Arthropods of the Tundra (NeAT).

Address: Department of Bioscience, Aarhus University, Grenåvej 14, 8410 Rønde, Denmark.

Liza K. Jenkins Ph.D. is a Research Scientist at the Michigan Tech Research Institute, at Michigan Technological University. Her research interests include landscape ecology, land cover change, and disturbance monitoring using remote sensing in high northern latitudes.

Address: Michigan Tech Research Institute (MTRI), Michigan Technological University, 3600 Green Court, Suite 100, Ann Arbor, MI 48105, USA.

e-mail: tth@bios.au.dk

Virve Ravolainen Ph.D. is a Scientist and a member of the vegetation expert network within CBMP. She works at the Norwegian Polar Institute and is responsible for vegetation monitoring and research in Svalbard within the Climate Ecological Observatory for Arctic Tundra-COAT program. Her research interests include tundra ecosystem change, with a focus on vegetation, and climate and herbivore impacts on it.

Address: Norwegian Polar Institute, Fram Centre, Postbox 6606, Langnes 9296, Troms $\varnothing$, Norway.

e-mail: virve.ravolainen@npolar.no

Paul A. Smith is a Research Scientist for Environment and Climate Change, Canada, and a member of the CBMP terrestrial steering group.

Address: Environment and Climate Change Canada, 1125 Colonel By Drive, Ottawa, ON K1A 0H3, Canada.

e-mail: paulallen.smith@canada.ca 
Pawel Wasowicz is a Research Scientist at the Icelandic Institute of Natural History and curator at the herbaria AMNH and ICEL. His research interests include plant biogeography, taxonomy, and invasion ecology.

Address: Icelandic Institute of Natural History, Borgir Nordurslod, 600 Akureyri, Iceland.

e-mail:pawel@ni.is
Niels Martin Schmidt Ph.D. is a Senior Scientist and member of the Terrestrial Expert Monitoring Group within CBMP and lead guest editor on the present special issue. He has been involved in the BioBasis Program running at Zackenberg and Nuuk for more than 20 years. His research mainly focuses on biotic interactions in a rapidly changing Arctic.

Address: Arctic Research Centre, Department of Bioscience, Aarhus University, Frederiksborgvej 399, 4000 Roskilde, Denmark.

e-mail:nms@bios.au.dk 